\title{
Bayesian Models for the Determination of Resonant Frequencies in a DI Diesel Engine
}

\author{
Timothy Bodisco*, ${ }^{*}$, Robert Reeves ${ }^{2}$, Rong Situ $^{3}$, Richard Brown ${ }^{1}$ \\ ${ }^{1}$ Faculty of Built Environment and Engineering, Queensland University of Technology \\ ${ }^{2}$ School of Mathematical Sciences, Queensland University of Technology \\ ${ }^{3}$ School of Engineering and Physical Sciences, James Cook University
}

\begin{abstract}
A time series method for the determination of combustion chamber resonant frequencies is outlined. This technique employs the use of Markov-chain Monte Carlo (MCMC) to infer parameters in a chosen model of the data. The development of the model is included and the resonant frequency is characterised as a function of time. Potential applications for cycle-by-cycle analysis are discussed and the bulk temperature of the gas and the trapped mass in the combustion chamber are evaluated as a function of time from resonant frequency information.
\end{abstract}

Key words: Resonant Frequency, MCMC, Inter-cycle Variability, Statistical Inference

\section{Introduction}

Calculating the resonant frequency accurately in an engine combustion chamber enables the characterisation of resonant frequencies that are associated with the speed of sound, and hence temperature $[3,4,5,6,7]$. Therefore, accurate isolation of resonant frequencies and their decay, as a function of time, or crank-angle, will allow the bulk temperature of the gas in the combustion chamber to be determined at any point within the region of interest. Further, an investigation into these frequencies will allow for cycle-by-cycle

\footnotetext{
*Corresponding Author.

e-mail: timothy.bodisco@qut.edu.au
} 


\begin{tabular}{|c|c|}
\hline \multicolumn{2}{|l|}{ Nomenclature } \\
\hline Posterior & $\begin{array}{l}\text { A probability distribution that summarises } \\
\text { information about a random variable, or } \\
\text { parameter, after information from } \\
\text { empirical data is obtained[1]. }\end{array}$ \\
\hline Prior & $\begin{array}{l}\text { Information known about a parameter } \\
\text { before empirical information is obtained. }\end{array}$ \\
\hline Uninformative Prior & $\begin{array}{l}\text { A prior that assumes no information was } \\
\text { known about a particular parameter before } \\
\text { obtaining empirical information. }\end{array}$ \\
\hline Markov-chain & or MCMC is a set of computational \\
\hline Monte Carlo & $\begin{array}{l}\text { methods for sampling from probability } \\
\text { distributions. }\end{array}$ \\
\hline Gibbs Sampler & $\begin{array}{l}\text { A particular MCMC method to generate } \\
\text { predictive distributions. }\end{array}$ \\
\hline DIC & $\begin{array}{l}\text { The deviance information criterion, a } \\
\text { relative goodness of fit measure from the } \\
\text { posterior expectation of the deviance and } \\
\text { the effective number of parameters as a } \\
\text { measure of complexity }[2] \text {. }\end{array}$ \\
\hline Posterior Predictive & The mean value of each modeled \\
\hline Mean & observations posterior distribution. \\
\hline Precision & $\begin{array}{l}\text { A term applied to the likely spread of } \\
\text { estimates of a parameter in a statistical } \\
\text { model. }\end{array}$ \\
\hline Top Dead Centre & $\begin{array}{l}\text { When the piston is at the highest possible } \\
\text { location in the cylinder. Often denoted by } \\
\text { a crank-angle of } 0 \text { or } 360 \text {. }\end{array}$ \\
\hline
\end{tabular}


analysis to be conducted to investigate inter-cycle variability. The isolation of resonant frequencies also has important implications in the detection of knock[5, 8]. Moreover, instantaneous resonant frequency information is of more practical use in the elimination of knock than more easily measured factors, such as the rate of pressure rise[5].

Conventional spectral analyses, such as those carried out a by Fourier transform, fail to accurately interpret the resonant frequencies precisely if the resonance is non-stationary[5]. A common approach to get around non-stationary frequencies is the use of the Wigner-Ville Spectrum[4, 5, 8, 9, 10, 11, 12]. We propose, however, the use of Bayesian statistical inference which allows us to configure precisely a model for the observable information of interest. Markov-chain Monte Carlo (MCMC) using Gibbs Sampling is a statistical inference technique that can be used for parameter estimation in Bayesian statistical models and is the method employed in this paper[13]. Using this methodology has the advantage that it requires the user to state explicitly any assumptions being made in the calculation[14]. Hence, the user always knows exactly what problem is being solved. Our models are defined and fitted using the WinBUGS software package[15].

Our technique for isolating resonant frequencies specifies an analytic form for the signal, then uses MCMC to estimate each model parameter. In our example of engine data, from in-cylinder pressure readings, we seek to obtain frequency as a function of time, and hence observe it in terms of a crank-angle.

A further advantage of Bayesian statistical inference is that unlike other techniques[16] such as FFTs[17] or maximum entropy spectral analysis[18, 19], which require a battery of data, it works effectively on a single cycle. This eliminates the need for ad hoc methods such as cycle averaging or spectrum averaging to extract useful information - not to speak of excessive laboratory time collecting data. Variations from cycle-to-cycle can also be explored by performing analysis on each cycle completely independently of the others.

\section{Experimental Configuration}

Experiments were conducted on a naturally aspirated 4-cylinder Ford direct injection diesel engine $(2701 \mathrm{C})$. The engine has a capacity of $4.152 \mathrm{l}$, a bore of $108.2 \mathrm{~mm}$, a stroke length of $115 \mathrm{~mm}$, a compression ratio of 15.5 and max- 
imum power of $48 \mathrm{~kW}$ at $2500 \mathrm{RPM}$. The engine was coupled to a Froude DPX type Hydraulic Dynamometer with load applied by increasing the flow rate of water inside the dynamometer housing. In-cylinder pressure was measured with a PCB 112B11 piezoelectric transducer with a Data Translation (DT9832) simultaneous analogue to digital converter connected to a desktop computer running National Instruments LabView. Data was collected at a sample rate of 200,000 samples per second. During testing the engine was run on diesel fuel at 2000 RPM on full load. For a more detailed overview of the experimental setup, including emissions results, refer to the corresponding paper by Surawski et al. [20].

\section{Model Development}

In this section we illustrate the process of model building for this problem by introducing more complex model specifications in an orderly sequence. At each step we compare an index of model fit, the deviance information criterion (DIC)[21], to ensure that over-fitting has not occurred, and that an increase in complexity results in a better predictive model. The DIC is a relative measure of model fit; a decrease in DIC indicates an improvement in fit from the previous model. We also compare observed data to the posterior predictive mean to assess whether the model is capable of tracking important changes in the observed data. Figure 1 shows the signal that is the subject of this investigation.

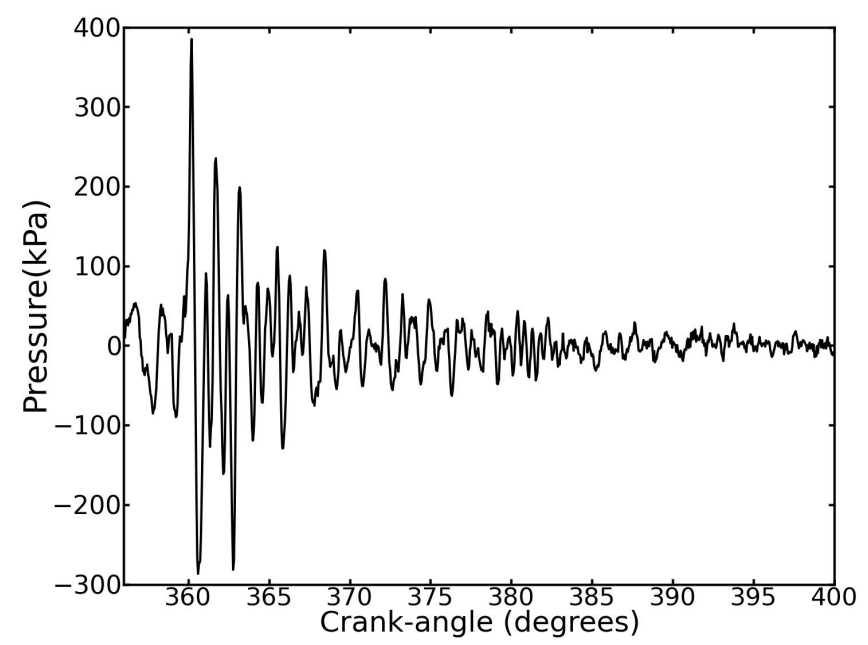

Figure 1: Pressure signal with the motoring frequency information removed 
Although it is not necessary to separate the analysis into the following steps it is more convenient, and less time consuming, to fit simpler models. Starting with a very simple model to observe what results can be obtained, a basic three parameter model is tested:

$$
\begin{aligned}
p(t) & \sim & N\left(\mu(t), \tau_{1}\right) \\
\tau_{1} & \sim & \operatorname{Gamma}(0.01,0.01) \\
\mu(t) & = & A \sin (\omega t+\phi) .
\end{aligned}
$$

The signal, $p(t)$, is modeled around a normally distributed time varying mean, $\mu$, with a sinusoidal characteristic. Here, and subsequently, we parameterise the Normal density with a precision parameter, $\tau$ where precision $=\frac{1}{\text { variance }}$, and give it an uninformative Gamma prior. This model attempts to fit a static amplitude and frequency to the signal. $\phi$ is given a uniform prior between $-\pi$ and $\pi, A$ is given an uninformative Normal prior and $\omega$ is given a uniform prior between 5000 and $7500 \mathrm{~Hz}$. The output from this model gives a DIC of 9505, and a posterior expectation of $\omega$ as $5960 \mathrm{~Hz}$. Visual inspection of the posterior predictive mean compared with the signal (Figure 2) tells us that this model does not adequately explain the observed changes.

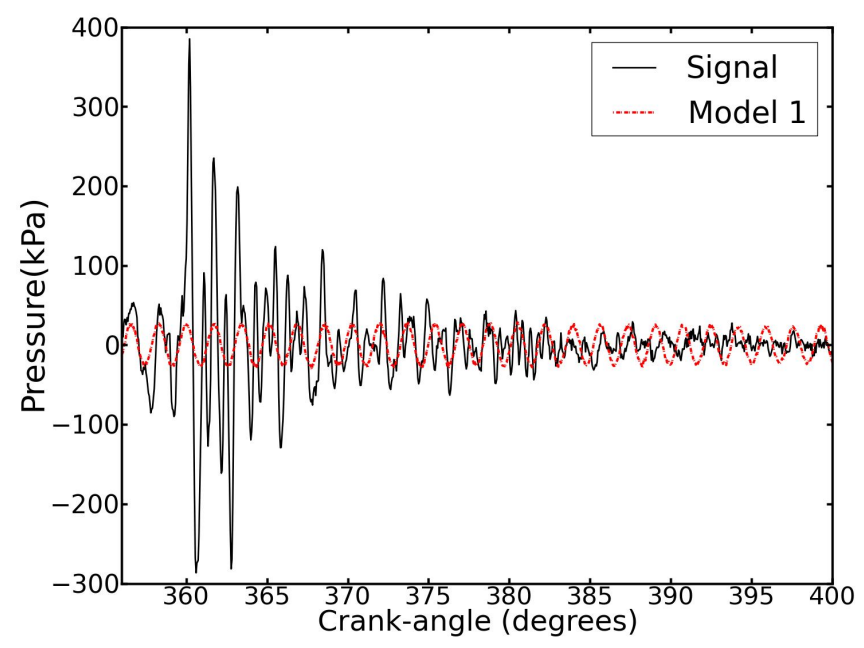

Figure 2: Model 1 plotted with the signal 
Including a term to model the decay in the amplitude seems to be a logical extension. Thus, we modify the model:

$$
\mu(t)=A e^{-\lambda t} \sin (\omega t+\phi),
$$

giving $\lambda$ an uninformative Normal prior. As expected there is a significant improvement in the model fit with a DIC now of 9362. Using this model the posterior expectation for $\omega$ is now $5994 \mathrm{~Hz}$. This is nearing our estimate, from a classical approach using FFTs (as shown later in Figures 8 and 9) of $6000 \mathrm{~Hz}$. Figure 3 shows the model fit.

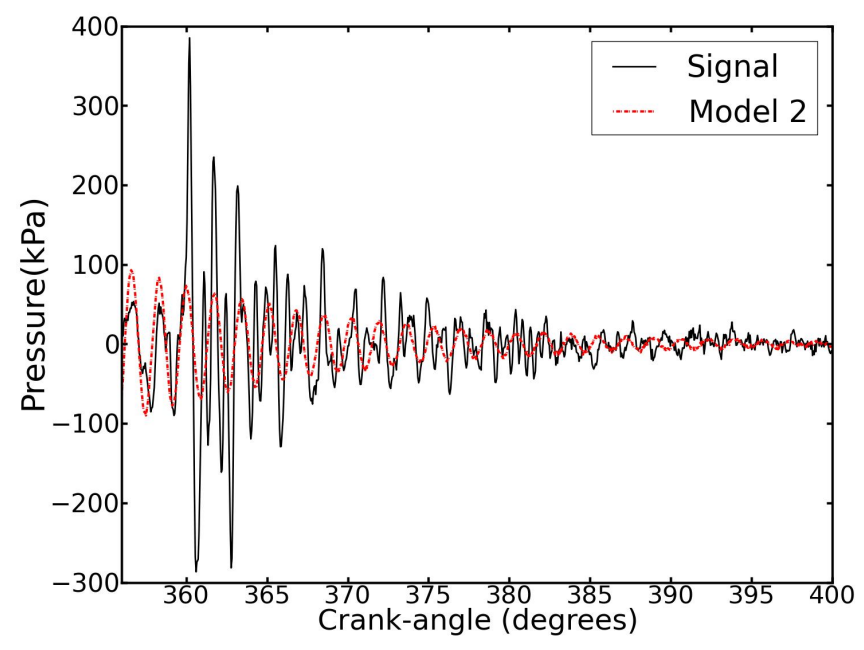

Figure 3: Model 2 plotted with the signal

This model fails to capture significant observed behaviour. However, given that it is known that resonant frequencies decay over time $[5,8,22]$ it seems intuitive to include a parameter that models the frequency decay. Hence, we extend the model by defining:

$$
\mu(t)=A e^{-\lambda t} \sin \left(\omega_{0} e^{-a t} t+\phi\right),
$$

setting $a$ to have an uninformative Normal prior. In this model, and thereafter, $\omega_{0}$ represents the initial first circumferential mode resonant frequency 
- from here out referred to as the resonant frequency. This model does not have a large effect on the deviance but, from a physics perspective, it is an important parameter - particularly from the aspect of what is desired from the model. It also stops the model from under-predicting the resonant frequency under the assumption that it is a stationary frequency. The minimal gain in the DIC, 9360, can be attributed to the very small decay in the resonant frequency, therefore providing evidence that there is minimal change in the resonant frequency as the crank-angle increases. Updated posterior expectations of our parameters are now $6107 \mathrm{~Hz}$ for $\omega_{0}$ and $2.1962 \times 10^{-10}$ for $a$, with our estimate for the resonant frequency now:

$$
\omega(t)=6107 e^{-2.1962 \times 10^{-10} t} .
$$

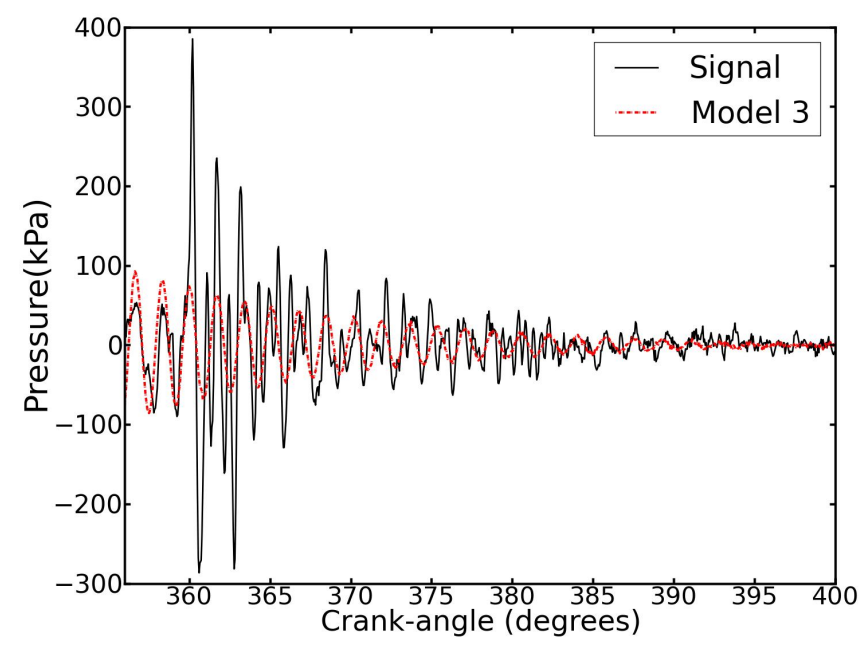

Figure 4: Model 3 plotted with the signal

However, comparing the posterior predictive mean to the observed signal (Figure 4) indicates multiple frequencies exist. Additional terms can be added to this, making the next model:

$$
\mu(t)=\sum_{i=1}^{3} A_{i} e^{-\lambda_{i} t} \sin \left(W_{i} \omega_{0} e^{-a_{i} t} t+\phi\right) .
$$


This will fit three different frequencies to the observed data. In this particular model, $W_{1}$ is set at 1 and $W_{2}$ and $W_{3}$ are given uniform priors between 1.5 and 2.5 , and 2.5 and 3.5 respectively. These priors were chosen to represent further higher frequency information that is present in the signal. The higher frequencies can be observed in an FFT (Figures 8 and 9). It is important that they do not overlap to avoid label switching and problems with convergence. Running this model yields a DIC of 9114, showing a significant improvement over the previous models. $\omega_{0}$ is now estimated to be $6097 \mathrm{~Hz}$ with the exponential decay constant $a$ estimated to be $2.073 \times 10^{-10}$. The resonant frequency is then estimated to be:

$$
\omega(t)=6097 e^{-2.073 \times 10^{-10} t} .
$$

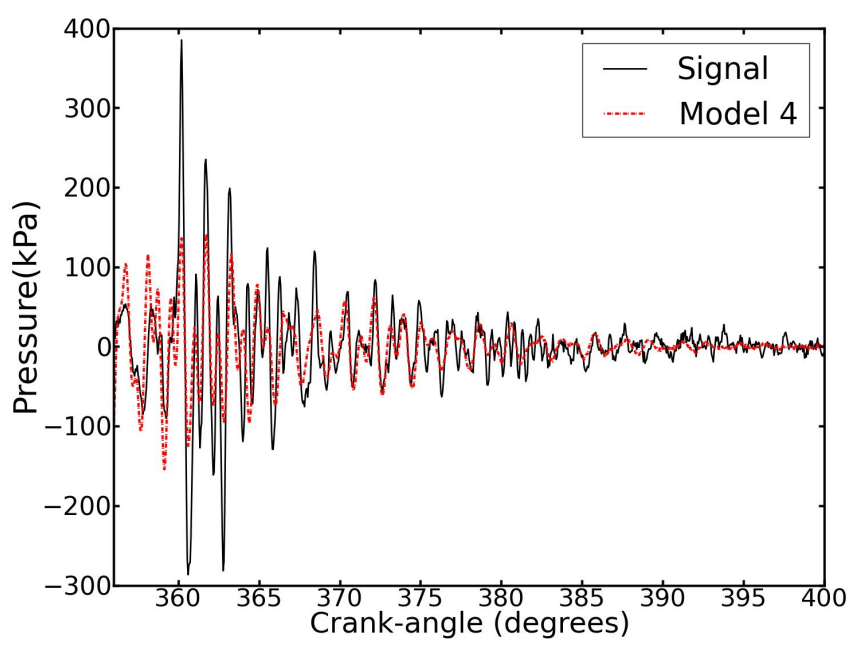

Figure 5: Model 4 plotted with the signal

Figure 5 shows that the model does not account for the sharp rise in the signal around top dead centre. Examination of Figure 1 gives an indication that the higher frequencies tend to occur somewhere around the peak. The inclusion of a step function in the non-fundamental terms may be a possible solution. Therefore, the following model is suggested: 


$$
\begin{aligned}
& f_{i}(t)=A_{i} e^{-\lambda_{i} t} \sin \left(W_{i} \omega_{0} e^{-a_{i} t} t+\phi\right) \\
& \mu(t)=f_{1}(t)+H(t-\delta) \sum_{i=2}^{3} f_{i}(t),
\end{aligned}
$$

where $H(t-\delta)$ is a step function where $H(t-\delta)=0$ for $t<\delta$ and $H(t-\delta)=1$ for $t \geq \delta$.

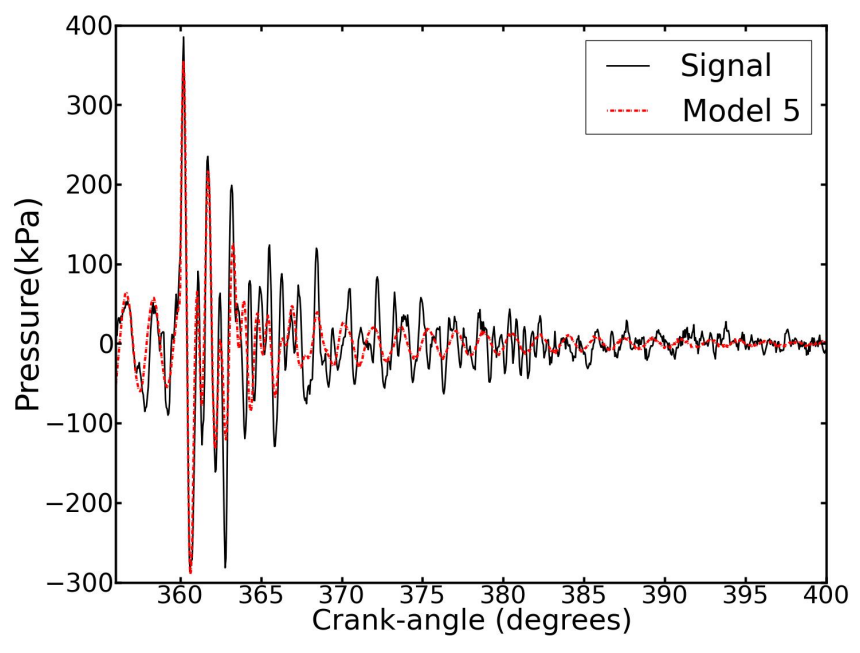

Figure 6: Model 5 plotted with the signal

Figure 6 shows that this method of compensating for the start of the nonfundamental frequencies has returned a very significant improvement in model fit (indicated by the improvement of the DIC to 8658). Now, $\omega_{0}$ is $6076 \mathrm{~Hz}$ with the exponential decay constant $a$ being $1.686 \times 10^{-10}$ estimating the resonant frequency as a function of time:

$$
\omega(t)=6076 e^{-1.686 \times 10^{-10} t} .
$$

Further parameters to fit this data are unnecessary and could be counter to the aim of the model; the DIC penalises the use of too many parameters as there is the risk of over fitting the data. Also, with more parameters there is increased concern that a lack of convergence leading to probability density 
functions (pdf) that are very multi-modal and hence a poor indicator of the desired result. Multi-modal pdf's can also occur as a result of label switching, especially with models where the parameters are heavily dependent on each other, such as in this case. Computationally, it is also wise to avoid over fitting the data with excessive parameters.

A posterior density plot of $\omega_{0}$ (Figure 7 ) and a plot of a fast Fourier transform (FFT) (Figures 8 \& 9) indicate the similarity. Note that the Bayesian method not only accurately describes the resonant frequency and its decay, but also it gives us the uncertainty in the parameter estimates, which is greatly improved when compared to the traditional method. Further, the FFT method assumes stationary frequency components, whereas our approach allows for the inclusion of decaying frequency components, which physical knowledge of the situation suggests are present.

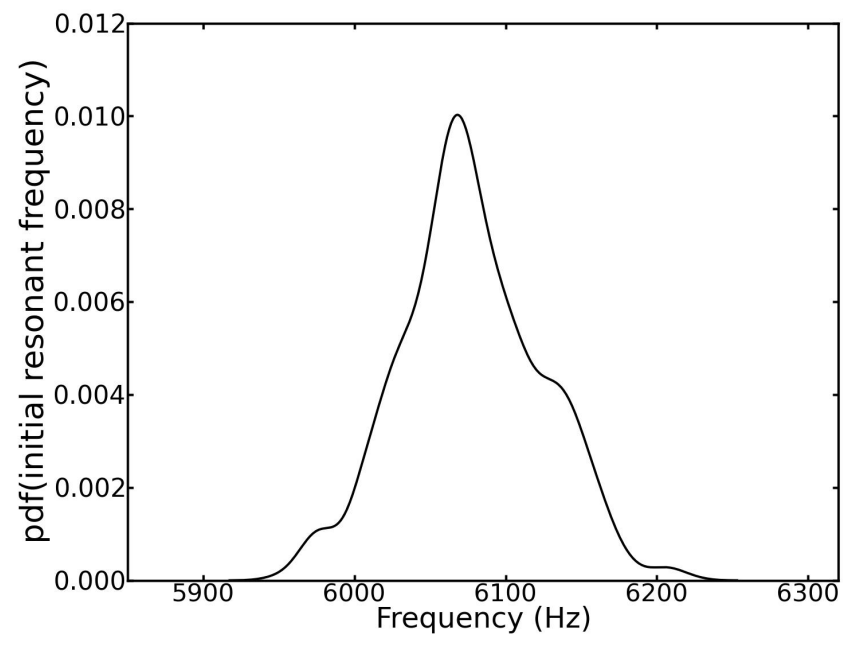

Figure 7: Posterior density of the initial resonant frequency 


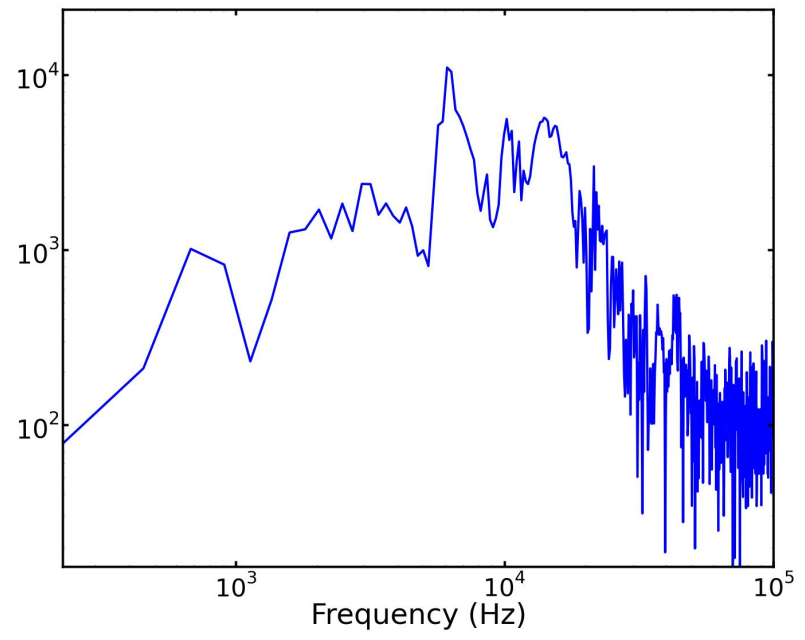

Figure 8: Fast Fourier transform of the region of interest

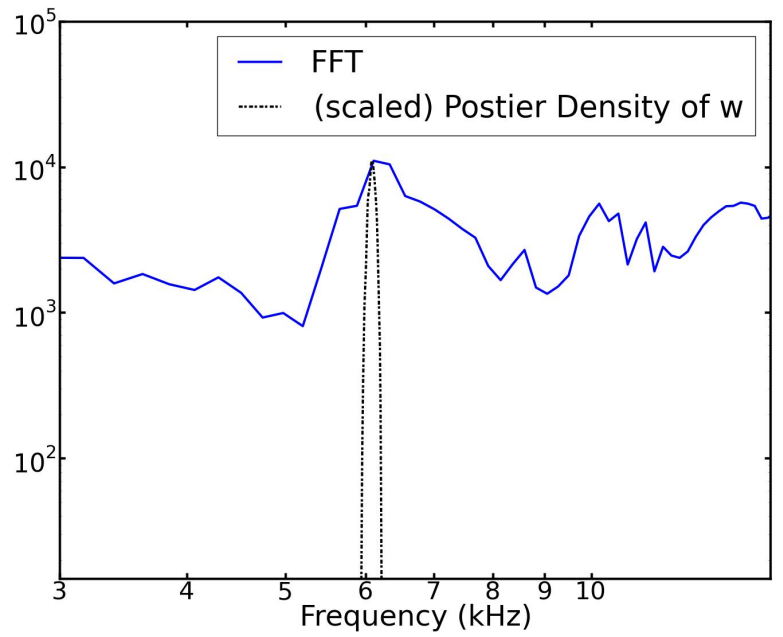

Figure 9: Comparison between Figure 7 and a Fast Fourier transform of the region of interest

The original intention in developing this model was to determine the resonant frequency as a function of time, or a crank-angle. This relationship is given 
in equation 6 and shown in Figure 10 along with the third circumferential mode resonant frequency information that was also calculated. Our findings support the results obtained in [22], which show using time-frequency analysis that the decay in the resonant frequencies are marginal. The similarity of the decay of both resonant frequencies is an indication that the model is working well, from a theoretical stand-point the decay rates should be equal. The difference is attributable to the start positions, the third circumferential term is prefixed by a step function, and because the decay for both modes are calculated independently.

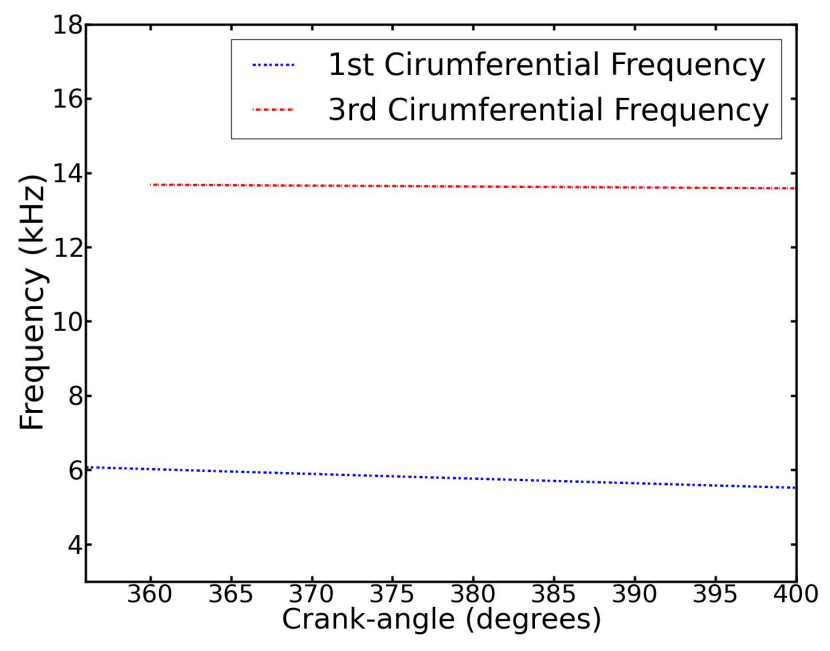

Figure 10: Drop in resonant frequency as a function of crankangle. Taken from the final model (model 5)

\section{Cycle-by-cycle Analysis}

Applying model 5 across a range of cycles demonstrates the importance of using such a powerful inferential technique in this type of data analysis. Figure 11 shows the pdfs of many cycles taken from the same data set, showing the range of inter-cycle differences. Also visible are some cycles with lower resonant frequencies - these have been attributed to misfires and ignition delay. 


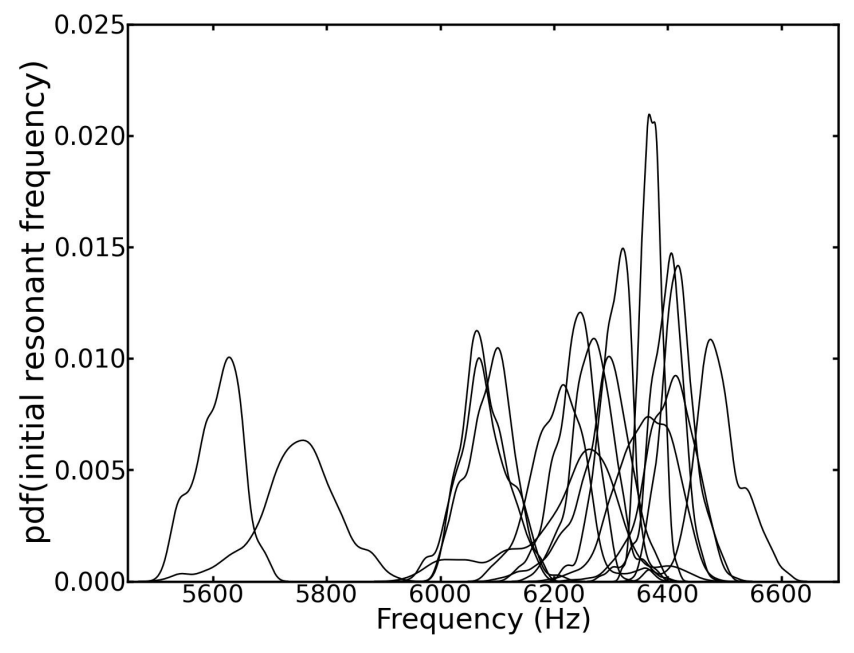

Figure 11: Probability density functions of the initial resonant frequency across a range of cycles

The cycle-to-cycle variation that can be seen highlights the reason that ad hoc techniques such as cycle averaging or frequency spectrum averaging are inappropriate for conducting frequency analysis with internal combustion engines. The subtle information contained in these higher frequencies will be skewed, or removed, by cycle averaging; hence, eliminating the point and usefulness of spectral analysis when applied to locating the resonant frequency from the in-cylinder pressure.

If the assumption of homogeneous composition of the control volume is assumed then the resonant frequency is related to the speed of sound and hence the temperature:

$$
T=(\gamma R)^{-1}\left(f B / \alpha_{m n}\right)^{2},
$$

where $T$ is temperature, $\gamma$ is the ratio of the specific heat of the gas, $R$ is the characteristic gas constant, $f$ is the resonant frequency, $B$ is the bore size and $\alpha_{m n}$ is the non-dimensional number. This relationship between the resonant frequency and the temperature allows research into another facet of combustion phenomena. 
Cycle-by-cycle analysis of the resonant frequency can yield interesting insights into the consistency of the combustion process. The relationship between frequency and temperature $\left(T \propto f^{2}\right)$ allows us to infer changes in combustion temperature and subsequently draw conclusions - such as attributing the lower frequency pdfs in Figure 11 to misfires and ignition delay. Investigating the spread of the most likely estimate of these frequencies (the modal point) can be used both as a method to make judgments on the similarity of each combustion and as a vehicle for comparing operating conditions of an engine. Applications for this could be on-going condition monitoring or the evaluation of alternative fueling strategies.

\section{Trapped Mass}

Temperature of the gas in the combustion chamber, as a function of time, gives an indicator of what is occurring during combustion and the thermodynamic processes in the cylinder of the engine[3]. Equation 7 can be used to calculate the bulk temperature of the gas in the combustion chamber as a function of time, or crank-angle, using the resonance information found from model 5. Thus, providing a method of determining this information from a standard engine testing laboratory. Values for $\gamma$ and $R$ are taken from AVL Boost simulations and the non-dimensional number $\alpha_{m n}$ can be calculated by solving the equation:

$$
J_{m}^{\prime}\left(\pi \alpha_{m n}\right)=0,
$$

where $J_{m}^{\prime}$ is the derivative of the Bessel function of the first kind of order $m[3,23]$.

$$
\begin{aligned}
& \alpha_{1,0}=0.5861 \\
& \alpha_{3,0}=1.3373
\end{aligned}
$$

Temperature, which is relatively constant at $3200 \mathrm{~K}$, can be used to estimate the trapped mass in the cylinder using the ideal gas relationship $P V=m R T$, hence:

$$
m(t)=\frac{P(t) V(t)}{R T(t)}
$$

where $P(t)$ and $V(t)$ are the experimentally measured pressure and volume time-series. Therefore, the trapped mass estimate as a function of crankangle is in Figure 12. 


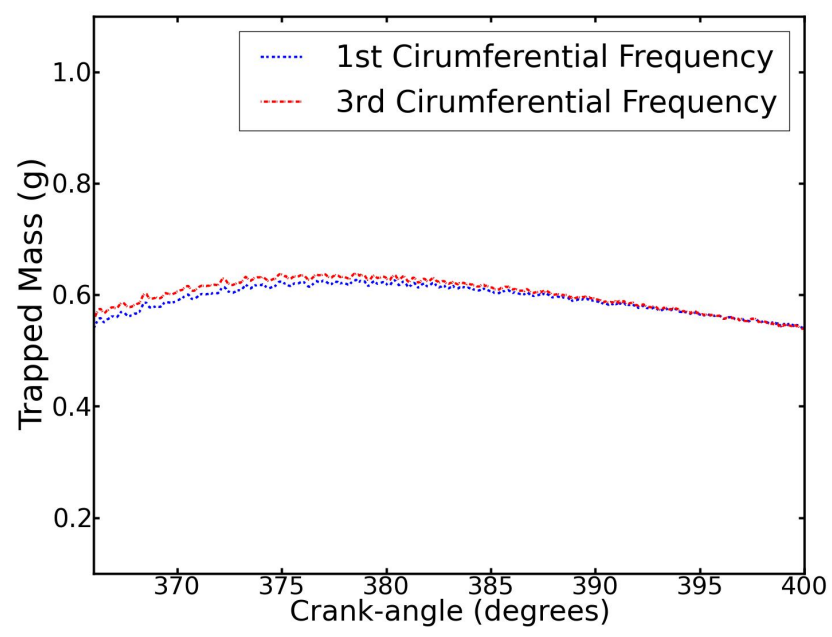

Figure 12: Estimated trapped mass calculated from combustion resonance and the ideal gas law

\section{Limitations}

The main limitation of this method is the problem of convergence. If the model used does not fit the data well then it is possible that results will not converge to any particular result. This makes model selection very application dependent. In this instance, the desired application was to demonstrate the use of this method to obtain very specific frequency information from a cycle. Thus, the final model was very specific.

Having a very specific model also has a limitation in an application such as this in that should the data present itself in a manner that deviates from the expected, the model may no longer fit the data well and useful information may no longer be gained from it. Therefore, if the goal is to analyse every cycle across a period of time to investigate true cyclic variability it suggested that simpler models be used that still find the desired information. In this instance, the use of model 3 would be a reasonable choice. The model is simple and hence more likely to converge with subtle changes in data, while still capturing the desired information well. Figure 13 shows the pdfs for the initial resonant frequency predicted by each model. 


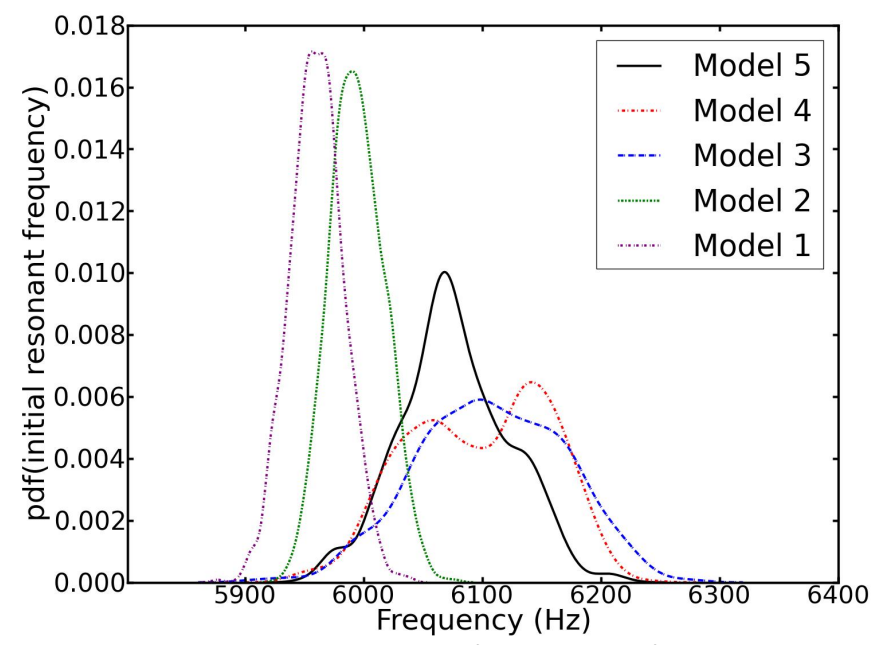

Figure 13: Probability density functions for the initial resonant frequency from models 1 to 5

It can be seen that models 1 and 2 give significantly different pdfs compared to the later models. This is to be expected as neither model allows the frequency to decay, and hence will return a result similar to that of the FFT which makes the same assumption. However, models 3 and 5 return similar information, with the difference being that model 5 has less uncertainty due to better model fit. This makes model 3 an ideal choice for model selection if many cycles in sequence are to be analysed and there are problems with cyclic changes that cause non-convergence.

\section{Further Work}

Further work will focus on a modern Cummins $5.9 l$ turbo-charged diesel engine running on neat diesel fuel and also with diesel ethanol combinations (blended and fumigated) and other alternative fuels, such as LPG. It is proposed that, under various fueling strategies the engine will exhibit different cyclic behaviour, with respect to the resonant frequency, which will aid in the evaluation of alternative fuels and help us to further understand the phenomena of combustion of diesel engines in dual-fuel operation.

Applications of this method of analysis extend further than being able to identify resonant frequency information. With more sophisticated models that capture the initial rise in temperature at the onset of combustion investigations involving trapped mass could be done to experimentally determine 
blow-by on a cycle-by-cycle basis. This type of analysis is relevant to signals taken from accelerometers to quantify knock in a manner that would allow comparisons between operating conditions. A further application could be the extraction of resonant frequency information directly from an accelerometer signal. Careful filtering would be required in this case.

\section{Conclusion}

This paper has introduced a powerful inference technique for the determination of resonant frequencies in a DI diesel engine which explicitly model the time dependence of the resonant frequency. This leads to a superior characterisation of important frequency behavior over FFT methods, which assume frequency components are time invariant, without introducing the complexities of other time-frequency analyses. Compared to results obtained from FFTs our method provides superior resolution and information about the time dependence of the resonant frequency. Our results provide a solid reason for the use of Bayesian inference as a method of analysing in-cylinder pressure data.

\section{Acknowledgments}

The authors wish to thank the following undergraduate students for dynamometer operation during experimental campaigns: Mr Adrian Schmidt, Mr Peter Clarke, Mr Yoaann Despiau and Mr Steven Herdy. We also wish to thank Mr Tony Morris for assisting with the design of experimental campaigns and Mr Jon James and Mr Glenn Geary for enabling an undergraduate teaching engine to be used for research purposes. Further thanks also to Technologist Mr Ken McIvor for his assistance in setting up the data acquisition software. Also, thanks to Ph.D student Mr Nicholas Surawski for his work in making the experiments a success. This work was undertaken under an Australian Research Council Linkage Grant (LP0775178) in association with Peak3 P/L.

\section{References}

[1] B. S. Everitt, The Cambridge Dictionary of Statistics, Third Edition, Cambridge University Press, 2006.

[2] A. Berg, R. Meyer, J. Yu, Deviance information criterion for comparing stochastic volatility models, Journal of Business \& Economic Statistics 22 (2002) 107-120. 
[3] R. Hickling, D. A. Feldmaier, F. H. K. Chen, J. S. Morel, Cavity resonances in engine combustion chambers and some applications, Acoustical Society of America Journal 73 (1983) 1170-1178.

[4] J. Bohme, D. Konig, Statistical processing of car engine signals for combustion diagnosis, in: Statistical Signal and Array Processing, 1994., IEEE Seventh SP Workshop on, 1994, pp. 369-374.

[5] Y. Ren, R. B. Randall, B. E. Milton, Influence of the resonant frequency on the control of knock in diesel engines, Proceedings of the Institution of Mechanical Engineers: Part D : Journal of Automobile Engineering. London 213 (2) (1999) 127-133.

[6] F. Payri, A. Broatch, B. Tormos, V. Marant, New methodology for incylinder pressure analysis in direct injection diesel engines: application to combustion noise, Measurement Science and Technology 16 (2) (2005) $540-547$.

[7] A. J. Torregrosa, A. Broatch, X. Margot, Combustion chamber resonances in direct injection automotive diesel engines: a numerical approach, International Journal of Engine Research 5 (1) (2004) 83-91.

[8] B. Samimy, G. Rizzoni, Mechanical signature analysis using timefrequency signal processing: application to internal combustion engine knock detection, Proceedings of the IEEE 84 (9) (1996) 1330-1343.

[9] L. Stankovic, A multitime definition of the wigner higher order distribution: L-wigner distribution, Signal Processing Letters, IEEE 1 (7) (1994) 106-109.

[10] L. Stankovic, A method for time-frequency analysis, Signal Processing, IEEE Transactions on 42 (1) (1994) 225-229.

[11] C. Wang, Y. Zhang, Z. Zhong, Fault diagnosis for diesel valve trains based on time-frequency images, Mechanical Systems and Signal Processing 22 (8) (2008) 1981-1993.

[12] J. Antoni, Cyclostationarity by examples, Mechanical Systems and Signal Processing 23 (4) (2009) 987-1036. 
[13] L. Tierney, Markov chains for exploring posterior distributions, The Annals of Statistics 22 (4) (1994) 1701-1728.

[14] G. L. Bretthorst, C. R. Smith, Bayesian analysis of signals from closelyspaced objects, in: R. L. Caswell (Ed.), Infrared Systems and Components III, Proc. SPIE 1050, 1989.

[15] D. Spiegelhalter, A. Thomas, N. Best, WinBUGS Version 1.2 User Manual, MRC Biostatistics Unit (1999).

[16] E. T. Jaynes, Bayesian spectrum and chirp analysis, in: C. Smith, D. Reidel (Eds.), in Proceedings of the Third Workshop on MaximumEntropy and Bayesian Methods (1983), Boston, 1987.

[17] J. W. Cooley, J. W. Tukey, An algorithm for the machine calculation of complex fourier series, Mathematics of Computation 19 (90) (1965) 297-301.

[18] J. Burg, Maximum entropy spectral analysis, in: D. Childers (Ed.), in Proceedings of the 37th Meeting of the Society of Exploration Geophysicists. Reprinted (1978) in Modern Spectrum Analysis, 1967.

[19] J. Burg, Maximum entropy spectral analysis, Ph.D. thesis, Stanford University (1975).

[20] N. C. Surawski, B. Miljevic, B. A. Roberts, R. L. Modini, R. Situ, R. J. Brown, S. E. Bottle, Z. D. Ristovski, Particle emissions, volatility, and toxicity from an ethanol fumigated compression ignition engine, Environmental Science \& Technology 44 (1) (2010) 229-235.

[21] D. J. Spiegelhalter, N. G. Best, B. P. Carlin, A. van der Linde, Bayesian measures of model complexity and fit, Journal of the Royal Statistical Society. Series B (Statistical Methodology) 64 (4) (2002) 583-639.

[22] L. Stankovic, J. F. Bohme, Time-frequency analysis of multiple resonances in combustion engine signals, Signal Processing 79 (1) (1999) $15-28$.

[23] P. M. Morse, K. U. Ingard, Theoretical Acoustics, McGraw-Hill Book Company, 1968. 